\title{
Aplicación del entorno 2.0 al estudio del fenómeno del lobby en el marco de la situación económica española
}

\author{
Juan Enrique GonZÁLvez VALLÉS \\ Universidad Complutense de Madrid \\ juanengo@ucm.es
}

\begin{abstract}
Resumen:
La campaña de imagen sobre las agencias de calificación fue llevada a cabo por un equipo docente e investigador de varias universidades españolas y extranjeras. Se trataba de plantear a la Opinión Pública Española y mundial el tema de las tres principales agencias de calificación desde dos puntos de vista opuestos que se solidificaron en dos campañas antitéticas de defensa de intereses contrapuestos sociales, ideológicos y, principalmente, económicos (llamadas lobby y contralobby). La primera fase busca los mecanismos del entorno 2.0 que reflejen la imagen de estas agencias, de modo holístico.
\end{abstract}

Palabras clave: Campaña; imagen; agencias de calificación; lobby; contralobby

\section{Application of the 2.0 environment to study the phenomenon of lobbying under the Spanish economic situation}

\begin{abstract}
:
The image campaign on the rating agencies was conducted by researchers and lecturers in several Spanish and foreign universities. It was raised to the Spanish and World Public Opinion theme of the three major rating agencies for two opposing views that solidified into two antithetical social advocacy campaigns, and ideological interests involved mainly economic (calls lobby and contralobby). The first phase seeks 2.0 environment mechanisms that reflect the image of these agencies, holistically.
\end{abstract}

Key Words: Campaign; image; rating agencies; lobby; contralobby

\section{Referencia normalizada:}

González Vallés, J. E. (2014): Aplicación del entorno 2.0 al estudio del fenómeno del lobby en el marco de la situación económica española. Historia y Comunicación Social. Vol. 19. Núm. Especial Marzo. Págs. 629-648.

Sumario: 1. Introducción. 2. Metodología. 3. Análisis y discusión. 4. Conclusiones. 5. Webgrafía. 


\section{Introducción}

La coyuntura económica que atraviesa España ha derivado en una serie de reacciones políticas y sociales que, desde el punto de vista tanto de la opinión pública, como de los actores conformantes de la misma, ha suscitado mucho interés. Además de las manifestaciones a la antigua usanza, ésas de pancarta y megáfono, tan propias de los años 80 y 90 del siglo pasado en nuestro país, ahora se está viviendo toda una revolución con la llegada de las nuevas tecnologías.

España no es ajena a la introducción de las redes sociales en este mundo globalizado y 2.0, y Facebook, Twitter y otras neuronas espejo han llegado a tener tanto impacto en la vida social del país como antaño lo conseguían los líderes de opinión a través de los medios convencionales. En este sentido, la crisis económica no estaba ajena a la opinión de los públicos dentro del panorama descrito anteriormente.

Han sido principalmente las agencias de calificación crediticia las que han venido a copar portadas de todos los medios por su labor antes y durante esta crisis que azota salvajemente a España. Sus criterios, su funcionalidad, su independencia y, sobre todo, su impacto sobre la micro y macroeconomía se han puesto en tela de juicio y han desatado una ola de reacciones en las diferentes redes sociales.

La opinión mayoritaria ha sido la de que su labor no ha sido la adecuada durante estas fases pero quedaba por contrastar empíricamente si esta sensación se plasmaba de forma clara a través de los canales de la Web 2.0. Por ello era necesario un estudio que planteara de forma equidistante esta cuestión y que, partiendo de una metodología concreta, específica y adecuada, llegara a ratificar esta afirmación o a refutarla.

Fruto de estas reflexiones surgió la campaña de imagen en la que se establecieron los grupos de presión que, fundamentalmente, tenían que trabajar en redes sociales para conocer la opinión pública española a este respecto. No se trataba de juzgar a nadie, pero sí se trataba de convencer a través de esta investigación a todos los que se quisieran acerca a ella de que podían decantarse por el grupo favorable a las agencias de calificación o al grupo en contra de las mismas, evaluando los elementos creativos $\mathrm{y} / \mathrm{o}$ informativos que se les presentaban.

\section{Metodología}

En primer lugar se realizó la elección del tema que se procedería a analizar, aunque el resultante fue la economía española, desde el punto de vista del análisis de la buena o mala imagen que poseía en el exterior y en el interior del país. Como esta temática era demasiado amplia y compleja como para abarcarla en una sola investigación, el acotamiento del objeto de estudio resultó imprescindible. Por eso lo reducimos al análisis del trabajo que realizan las agencias de calificación dentro de la economía española, ya sean éstos para instituciones públicas o privadas. 
Con el tema totalmente claro, los objetivos tenían que ser determinados, teniendo en cuenta que en la fase final de la campaña habría dos actores intervinientes, como son el Lobby, o grupo de presión; y el Contralobby, o grupo de presión contrario a los intereses del otro grupo. Todo ello focalizado hacia el seguimiento de los medios de comunicación y las redes sociales para aportar creatividades que apoyaran sus respectivas causas.

Pero la fase inicial de esta investigación consistía en una exhaustiva búsqueda de datos e información que ayudaran a los integrantes de los futuros grupos de presión a comprender en qué terreno iban a desarrollar sus campañas. A la manera del Usted Está Aquí de los mapas urbanos, los grupos se dividieron en departamentos para la obtención de una información holística y que cuyos segmentos eran: saber técnico, saber económico, saber jurídico y saber comunicacional, centrado especialmente en las campañas de imagen que se lanzaban a favor y en contra de las agencias de calificación.

Toda vez realizada esta primera fase, Lobby y Contralobby tomarían forma y realizarían su campaña de imagen. Tanto unos como otros debían proceder a emprender su campaña teniendo en cuenta que ésta empezaba en el mes de enero y desde ese momento hasta mayo la campaña se iba a desarrollar en redes sociales. Los dos grupos tenían que crear perfiles tanto en Facebook como en Twitter de forma obligatoria, así como en otras redes sociales si así lo estimaban oportuno. Durante estos meses los perfiles tenían que ser alimentados de forma continuada y los resultados debían ser entregados en forma de métricas detalladas cada semana.

La segunda parte dentro de esta segunda fase correspondía a la creación de un plan de comunicación que abarcara desde junio hasta el último día del año, donde ya había límites en cuanto a la distribución en medios, sino que podían planificar tanto en medios convencionales, televisión, radio y prensa, principalmente; como en nuevas tecnologías, especialmente la Web 2.0, con todos los medios promocionales que tiene adheridos. Para esta parte el presupuesto debía ser detallado y aprobado por los investigadores principales del grupo, aunque como referencia se les dotó de seis millones de euros para contratar espacios en los distintos medios de comunicación.

Posteriormente, a principios del mes de junio, ambos grupos de presión debían exponer tanto su trabajo durante cinco meses en las redes sociales como el plan de medios planificado hasta final de año. Se establecerían los términos de esta exposición mediante acuerdo entre los portavoces de los grupos, existiendo la posibilidad de ofrecer hasta dos réplicas a las exposiciones iniciales.

Tras la reunión de los coordinares de la investigación con los portavoces de los dos grupo, se pactó un turno de exposición de 20 minutos y ambos portavoces acordaron un turno de réplica de 10 minutos como tiempo máximo. Al no existir acuerdo para el orden de intervención se procedió al sorteo del mismo, quedando establecida la exposición del Contralobby en primer lugar y la del Lobby en segundo. Se indicó a ambos portavoces que este orden también se respetaría en el caso de la réplica y se les abordó para ofertar la posibilidad de una segunda réplica de hasta 5 minutos 
de duración. De nuevo no hubo acuerdo entre las partes, puesto que el Contralobby optaba por un segundo turno de réplica mientras que el Lobby no deseaba que éste tuviera lugar. Tras un nuevo sorteo a cara o cruz con una moneda, el azar decidió que no hubiera segundo turno de réplica, con lo que, tras el primer turno, quedaría cerrado el debate entre ambos grupos.

\section{Análisis y discusión}

\subsection{Resultados de los departamentos.}

Como ya explicitamos en la introducción, era nuestro propósito inicial el ofrecer un panorama completo de las distintas facetas que abarcan al término y a la realidad de las agencias de calificación. En casi tres meses de investigación, los distintos departamentos acumularon gran cantidad de información sobre el tema elegido proporcionando un informe final sobre su trabajo totalmente esclarecedor.

\subsubsection{Departamento técnico}

Las agencias de calificación, también denominadas de rating son organizaciones que evalúan determinados productos financieros y realizan calificaciones del riesgo de impago y solvencia basado en los activos de entidades, países, etc, pueden calificar la calidad de las deudas contraídas.

Se trata por tanto de empresas encaminadas a guiar las decisiones financieras de pequeños y grandes inversores pasando desde el particular hasta el Banco Central Europeo. Surgieron a principios del siglo XX vinculadas a las obligaciones de las empresas del sector ferroviario americano y han ido creciendo de manera notable por el desarrollo de los mercados financieros donde actualmente poseen una grandísima influencia.

La Comisión Europea publica de nuevo la lista en el Diario Oficial de la Unión Europea en el plazo de treinta días desde cualquier actualización, por lo que puede haber diferencias entre la lista publicada por la AEVM y la lista disponible en el Diario Oficial durante ese período. Fecha de la última actualización: 6 de enero de 2012 (durante el periodo de ejecución de esta investigación).

El mercado principalmente está en manos de tres compañías americanas:

- Standard \& Poor's.

- Moody's.

- Fitch Ratings.

Para realizar sus calificaciones se recogen los datos proporcionados por los países o las organizaciones para analizarlos y categorizar los resultados otorgándoles una 
nota dentro de sus escalas. Estas notas se clasifican en letras que van desde D para la puntuación más baja en Fitch o Standard \& Poors o la C en Moody's hasta la AAA, para las mayores puntuaciones.

\section{Gráfico 1. Rango de calificaciones a largo plazo}

\begin{tabular}{|c|c|c|c|c|}
\hline \multirow[t]{3}{*}{ Deuda largo plazo } & $\mathbf{S \& P} \mathbf{P}^{(1)}$ & MOODY'S (2) & $\mathrm{FITCH}^{(1)}$ & Significado \\
\hline & AAA & Aaa & AAA & Calidad crediticia óptima. \\
\hline & AA & $\mathrm{Aa}$ & AA & Alta calidad crediticia. \\
\hline \multirow{2}{*}{$\begin{array}{l}\text { Grado } \\
\text { Inversión }\end{array}$} & A & A & A & Buena calidad crediticia. \\
\hline & BBB & Baa & BBB & $\begin{array}{c}\text { Calidad crediticia satisfactoria. Existen } \\
\text { tensiones a largo plazo. Acontecimientos } \\
\text { adversos pueden empeorar su capacidad de } \\
\text { pago. }\end{array}$ \\
\hline \multirow[t]{3}{*}{$\begin{array}{l}\text { Grado } \\
\text { especulación }\end{array}$} & BB & $\mathrm{Ba}$ & BB & $\begin{array}{l}\text { Calidad crediticia cuestionable. Futuro } \\
\text { incierto pero con capacidad actual. }\end{array}$ \\
\hline & B & B & B & $\begin{array}{l}\text { Calidad crediticia pobre/dudosa. La capacidad } \\
\text { para hacer frente a las deudas en el largo } \\
\text { plazo es baja. }\end{array}$ \\
\hline & $\operatorname{ccc}$ & Caa & $\operatorname{ccc}$ & $\begin{array}{l}\text { Calidad crediticia muy pobre. Posibilidad } \\
\text { actual de algún tipo de impagos. }\end{array}$ \\
\hline
\end{tabular}

Gráfico 2. Rango de calificaciones a largo plazo (2)

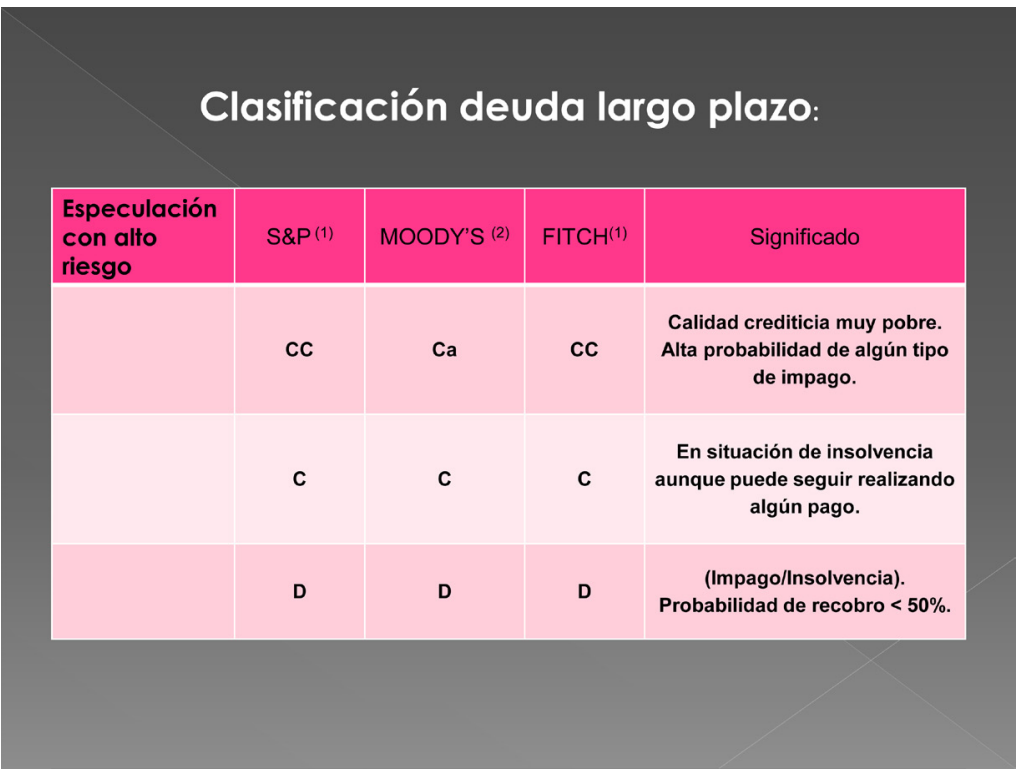


Gráfico 3. Rango de calificaciones a largo plazo (y 3)

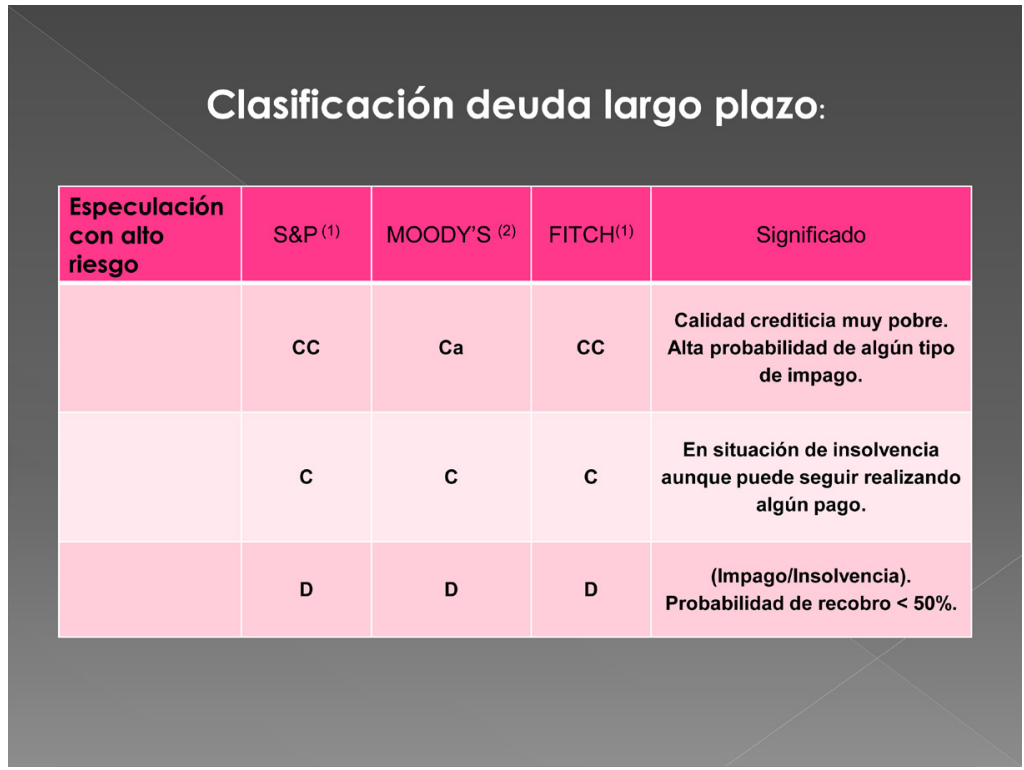

Gráfico 4. Rango de calificaciones a corto plazo (Standard \& Poors).

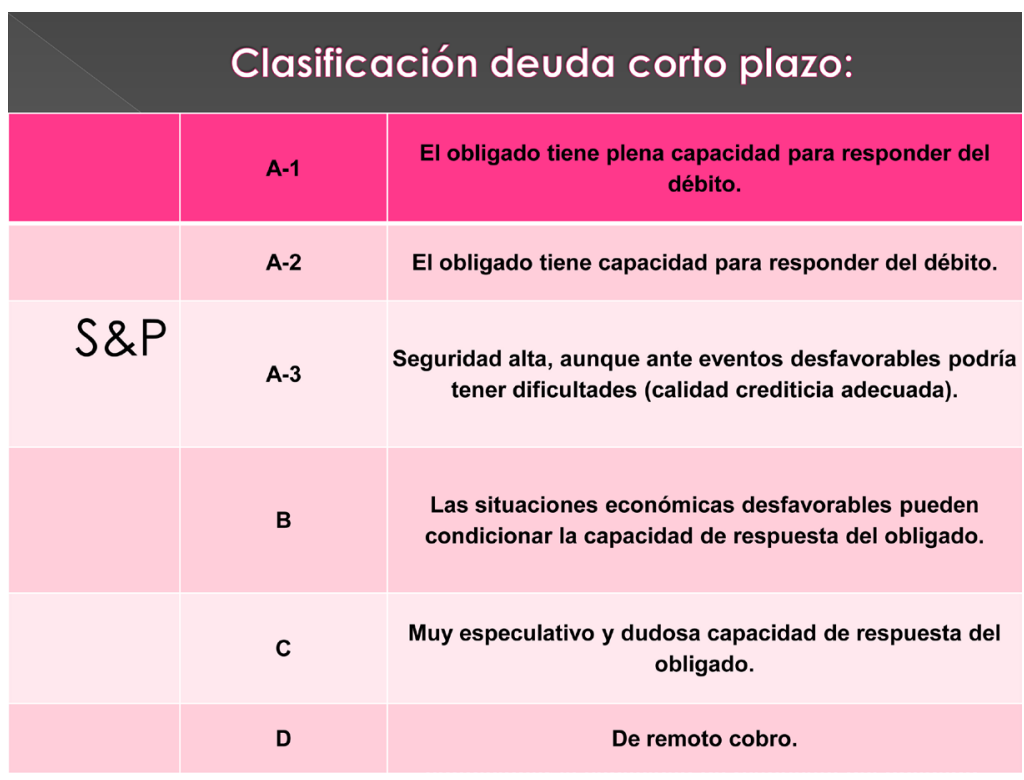


Gráfico 5. Rango de calificaciones a corto plazo (Moody's).

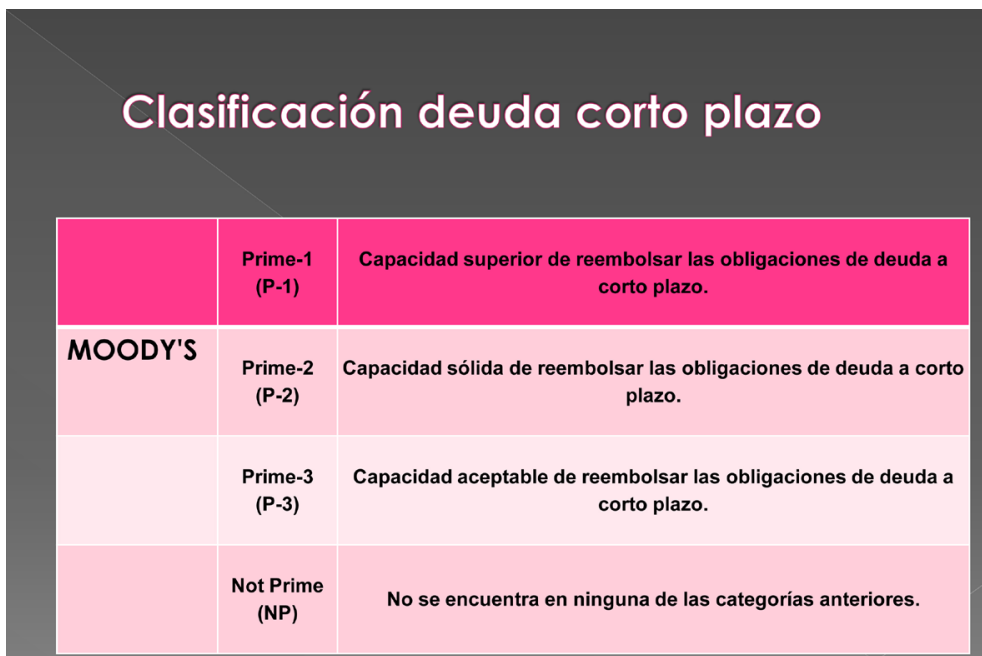

Gráfico 6. Rango de calificaciones a corto plazo (Ficht)

\section{Clasificación deuda corło plazo}

\begin{tabular}{|c|c|c|}
\hline & F-1 & $\begin{array}{l}\text { Máxima seguridad de pago (calidad crediticia máxima). Puede } \\
\text { Ilevar un signo positivo (+) para indicar una gran solvencia. }\end{array}$ \\
\hline FITCH & F-2 & $\begin{array}{r}\text { Seguridad muy alta y riesgo de impago bajo (calidad crediticia } \\
\text { buena). }\end{array}$ \\
\hline F-3 & $\begin{array}{r}\text { Seguridad alta, aunque ante eventos desfavorables podria tener } \\
\text { dificultades (calidad crediticia adecuada). }\end{array}$ \\
\hline B & $\begin{array}{r}\text { Capacidad muy justa para hacer frente a sus obligaciones } \\
\text { (especulativo). Hay que vigilar su evolución, ya que podría tener } \\
\text { problemas. }\end{array}$ \\
\hline C & Las posibilidades de impago son altas. \\
\hline & Emisores o emisiones con impagos. \\
\hline
\end{tabular}


Los principales clientes de las agencias de calificación son las siguientes:

Gráfico 7. Principales clientes de las agencias de calificación

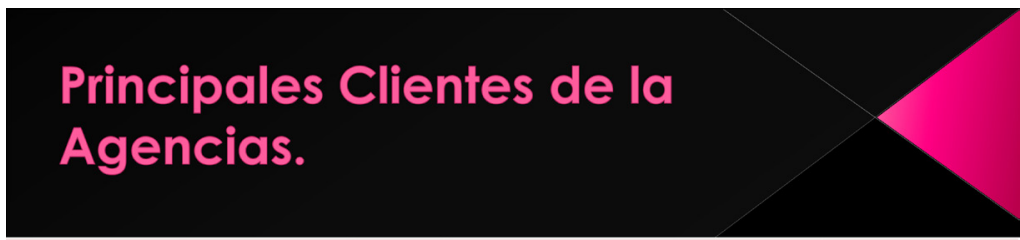

\begin{tabular}{|c|c|c|c|c|c|c|c|c|}
\hline \multirow{2}{*}{ Entidad Financiera } & \multirow{2}{*}{ C.B.P } & \multicolumn{4}{|c|}{ Ratings } & \multicolumn{3}{|c|}{ Test Estrés } \\
\hline & & Fitch L.P. = & Fitch C.P. ${ }^{*}$ & Moody's $=$ & $\operatorname{sep}=$ & 2009 & 2010 & 2011 \\
\hline - BNP Paribas & & AA & $\mathrm{F} 1+$ & $A 2$ & & & & \\
\hline - Cortal Consors & & $\Delta A$ & $\mathrm{~F} 1+$ & & & & & \\
\hline - Cetelem & & $A A$ & F1 & & & & & \\
\hline - ABN AMRO Bank & & AA- & $\mathrm{F} 1+$ & & & & & \\
\hline - Banco Popular-e & $\infty$ & AA- & $\mathrm{F} 1+$ & & & & & \\
\hline - Banco de Andalucía & & AA- & $\mathrm{F} 1+$ & & & & & \\
\hline - Barclays & $\infty$ & AA- & F1 & & BEB- & & & \\
\hline - Caixa Geral & $\infty$ & AA- & F1 & & & & & \\
\hline - Citibank & & A+ & $\mathrm{F} 1+$ & $A 3$ & B- & & & \\
\hline - ING Direct & & At & $\mathrm{F} 1+$ & $\mathrm{Aa3}$ & At & & & \\
\hline - Banco cooperativo & & A & F1 & & & & & \\
\hline - Cajasstur & $\infty$ & A- & F1 & $\mathrm{Baa} 2$ & & $7,8 \%$ & $8,3 \%$ & $6,8 \%$ \\
\hline - Caixa Nova & $\approx$ & A- & $\mathrm{F} 2$ & B1 & & $7.2 \%$ & $5,2 \%$ & $5,3 \%$ \\
\hline - Caja San Fernando & & A- & $F 2$ & & & & & \\
\hline - Caja de Extremadura & $\infty$ & A- & F2 & & & $7,8 \%$ & $8,3 \%$ & $6,8 \%$ \\
\hline - Bankinter & $\infty$ & $\mathrm{BBB}+$ & F1 & Ba1 & $\mathrm{BB}+$ & $6,8 \%$ & $6,2 \%$ & $5,3 \%$ \\
\hline - BBVA & $\infty$ & $\mathrm{BBB}+$ & $\mathrm{F} 2$ & $\mathrm{Baa} 3$ & BDB- & $9,3 \%$ & $8,0 \%$ & $9,2 \%$ \\
\hline - Banco Santander & $\infty$ & $\mathrm{BBB}+$ & $\mathrm{F} 2$ & Baa2 & B日B & $10,2 \%$ & $7,1 \%$ & $8,4 \%$ \\
\hline - Banesto & $\infty$ & $\mathrm{BBB}+$ & F2 & Ваaз & B日B & & & \\
\hline$-\mathrm{ccm}$ & & $\mathrm{BBB}+$ & F2 & & & & & \\
\hline - Caixa Catalunya & $\infty$ & $\mathrm{BBB}+$ & F2 & B1 & & $3,9 \%$ & $6,4 \%$ & $4,8 \%$ \\
\hline - Caixa Galicia & $\infty$ & $\mathrm{BBB}+$ & F2 & B1 & & $7,2 \%$ & $5,2 \%$ & $5,3 \%$ \\
\hline - Caika Sabadell & $\infty$ & $\mathrm{BB} \mathrm{B}+$ & F2 & & & $4,7 \%$ & $6,3 \%$ & $4,5 \%$ \\
\hline - Calca Terrassa & $\infty$ & $8 B 8+$ & $F 2$ & & & $4,7 \%$ & $6,3 \%$ & $4,5 \%$ \\
\hline - Caja España & $\infty$ & $\mathrm{BBB}+$ & F2 & Baa3 & & $5,6 \%$ & $8,2 \%$ & $7,3 \%$ \\
\hline - Ibanesto & $\sim$ & $\mathrm{BBB}+$ & $\mathrm{F} 2$ & $\mathrm{Baa} 3$ & BEB & & & \\
\hline
\end{tabular}

Gráfico 8. Principales clientes de las agencias de calificación

\section{Principales Clientes de la Agencias.}

\begin{tabular}{|c|c|c|c|c|c|c|c|c|}
\hline - Sa Nostra & 2 & $\mathrm{BB}+$ & $\mathrm{F} 2$ & & & $7,0 \%$ & $8,3 \%$ & $6,1 \%$ \\
\hline - Banco Mare Nostrum & $\theta$ & $\mathrm{BB}+$ & $\mathrm{F} 3$ & & & & $8,3 \%$ & $6,1 \%$ \\
\hline - Activo Bank & $\diamond$ & $\mathrm{BB}+$ & $B$ & Ba1 & $\mathrm{B}+$ & & $6,2 \%$ & $5,7 \%$ \\
\hline - Banco Guipuzcoano & $\checkmark$ & $\mathrm{BB}+$ & $\mathrm{B}$ & & $\mathrm{B}+$ & & $6,2 \%$ & $5,7 \%$ \\
\hline - Banco Herrero & $\diamond$ & $\mathrm{BB}+$ & B & Ba1 & $\mathrm{B}+$ & & $6,2 \%$ & $5,7 \%$ \\
\hline - Banco Popular & 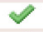 & $\mathrm{BB}+$ & $B$ & Ba1 & $\mathrm{BB}$ & $7,0 \%$ & $7,1 \%$ & $5,3 \%$ \\
\hline - Banco Sabadell & $\vartheta$ & $\mathrm{BB}+$ & B & Ba1 & $\mathrm{B}+$ & & $6,2 \%$ & $5,7 \%$ \\
\hline - Banco Urquijo & 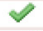 & $\mathrm{BB}+$ & B & Ba1 & $\mathrm{B}+$ & & $6,2 \%$ & $5,7 \%$ \\
\hline - Caja 3 & $\diamond$ & $\mathrm{BB}+$ & $B$ & & & & $8,6 \%$ & $4,0 \%$ \\
\hline - Liberbank & $\vartheta$ & $\mathrm{BB}+$ & $B$ & $\mathrm{Ba3}$ & & & $6,8 \%$ & $8,3 \%$ \\
\hline - NovaCaixaGalicia & $\diamond$ & $\mathrm{BB}+$ & B & $\mathrm{B1}$ & & & $5,2 \%$ & $5,3 \%$ \\
\hline - Sabadell Atlántico & $\curvearrowright$ & $\mathrm{BB}+$ & B & Ba1 & $\mathrm{B}+$ & $7,2 \%$ & $6,2 \%$ & $5,7 \%$ \\
\hline - Sabadell-CAM & $\leadsto$ & $\mathrm{BB}+$ & B & Ba1 & $\mathrm{B}+$ & $7,8 \%$ & $3,8 \%$ & $3,0 \%$ \\
\hline - Solbank & $\vartheta$ & $\mathrm{BB}+$ & B & $\mathrm{Ba} 3$ & $\mathrm{~B}+$ & & $6,2 \%$ & $5,7 \%$ \\
\hline - Unnim & $\diamond$ & $\mathrm{BB}+$ & B & & & $4,7 \%$ & $6,3 \%$ & $4,5 \%$ \\
\hline - Caja Círculo & 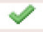 & $\mathrm{BB}+$ & & & & $6,1 \%$ & $8,6 \%$ & $4,0 \%$ \\
\hline - Evo Banco & $\infty$ & $\mathrm{BB}+$ & & B1 & & & $5,2 \%$ & $5,3 \%$ \\
\hline - NovaGalicia & $\vartheta$ & $\mathrm{BB}+$ & & B1 & & & $5,2 \%$ & $5,3 \%$ \\
\hline - Banco de Valencia & & BB- & B & B3 & & & & \\
\hline
\end{tabular}


Gráfico 9. Principales clientes de las agencias de calificación.

\section{Principales Clientes de la} Agencias.

\begin{tabular}{|c|c|c|c|c|c|c|}
\hline - BFS-Banco Finantia Sof & & & & & & \\
\hline - Banca March & $\vartheta$ & Baa3 & & $19,0 \%$ & $22,2 \%$ & $23,5 \%$ \\
\hline \multicolumn{7}{|l|}{ - Banco Alcalá } \\
\hline \multicolumn{7}{|l|}{ - Banco Base } \\
\hline \multicolumn{7}{|l|}{ - Banco Caminos } \\
\hline - Banco Espírito Santo & & & $\mathrm{BBB}$ & & & \\
\hline - Banco Etcheverría & $\vartheta$ & & & & & \\
\hline - Banco Gallego & $\vartheta$ & & & & & \\
\hline \multicolumn{7}{|l|}{ - Banco Madrid } \\
\hline - Banco Mediolanum & $\vartheta$ & & & & & \\
\hline - Banco Pastor & $\vartheta$ & Ba1 & & $6,0 \%$ & $7,6 \%$ & $3,3 \%$ \\
\hline \multicolumn{7}{|l|}{ - Banco Pichincha } \\
\hline \multicolumn{7}{|l|}{ - Banco de Crédito Balear } \\
\hline \multicolumn{7}{|l|}{ - Banco de España } \\
\hline - Banco de Madrid & & & & & & \\
\hline
\end{tabular}

Gráfico 10. Principales clientes de las agencias de calificación

\section{Principales Clientes de la Agencias.}

\begin{tabular}{|c|c|c|c|c|c|}
\hline \multicolumn{6}{|l|}{ - Bancofar } \\
\hline - Bancorreos & $\vartheta$ & & & & \\
\hline - Bankoa & 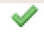 & Ba1 & & & \\
\hline \multicolumn{6}{|l|}{ - Bankpyme } \\
\hline \multicolumn{6}{|l|}{ - Bantierra } \\
\hline \multicolumn{6}{|l|}{ - Caixa Guissona } \\
\hline - Caixa Manlleu & $\checkmark$ & & $4,7 \%$ & $6,3 \%$ & $4,5 \%$ \\
\hline - Caixa Manresa & $\checkmark$ & B1 & $3,9 \%$ & $6,4 \%$ & $4,8 \%$ \\
\hline - Caixa Pollensa & $\checkmark$ & & $6,2 \%$ & & \\
\hline - Caixa Popular & $\checkmark$ & & & & \\
\hline \multicolumn{6}{|l|}{ - Caixa Rural Balears } \\
\hline - Caja Arquitectos & $\checkmark$ & & & & \\
\hline - Caja Caminos & 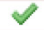 & & & & \\
\hline - Caja Cantabria & $\checkmark$ & Baa2 & $7,8 \%$ & $8,3 \%$ & $6,8 \%$ \\
\hline - Caja Duero & $\vartheta$ & Baa3 & $5,6 \%$ & $8,2 \%$ & $7,3 \%$ \\
\hline - Caja España-Duero & $\checkmark$ & & & $8,2 \%$ & $7,3 \%$ \\
\hline - Caja Guipuzcoa S.Sesbastián & & & $6,1 \%$ & & \\
\hline
\end{tabular}


Gráfico 11. Principales clientes de las agencias de calificación

\section{Principales Clientes de la Agencias.}

\section{Top 10 mejores bancos de noviembre según usuarios de tu capital}

\begin{tabular}{|c|c|c|}
\hline 1 & ING Direct & 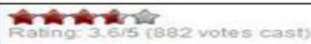 \\
\hline 2 & Activo Bank & 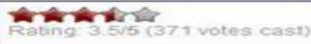 \\
\hline 3 & Evo Banco & Rating \\
\hline 4 & BankiaLink & RैAng \\
\hline 5 & Triodos Bank & 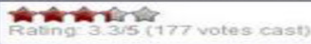 \\
\hline 6 & Sabadell Atlantico & Rating \\
\hline 7 & Banco Sabadell & 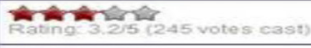 \\
\hline 8 & Ibanesto & Rating \\
\hline 9 & Bankinter & Rating $3.1 / 5$ (424 votes cast) \\
\hline 10 & Banco Popular & 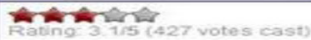 \\
\hline
\end{tabular}

Gráfico 12. Principales clientes de las agencias de calificación

\section{Principales Clientes de la Agencias.}

- Caja Insular de Canarias

- Caja Rural Intermediterranea

- Caja Rural de Burgos

- Caja Rural de Granada

- Caja Rural de Toledo

- Caja de Ingenieros

- CajaSiete

- Cajalon

- Cajas Rurales Unidas

- CatalunyaCaixa

- Deutsche Bank

- Halifax

- ICBC

- Ibercaja

- Inversis Banco

- Ipar Kutxa

- Lloyds Bank

- Multicaja

- Novanca

- O ficina Directa

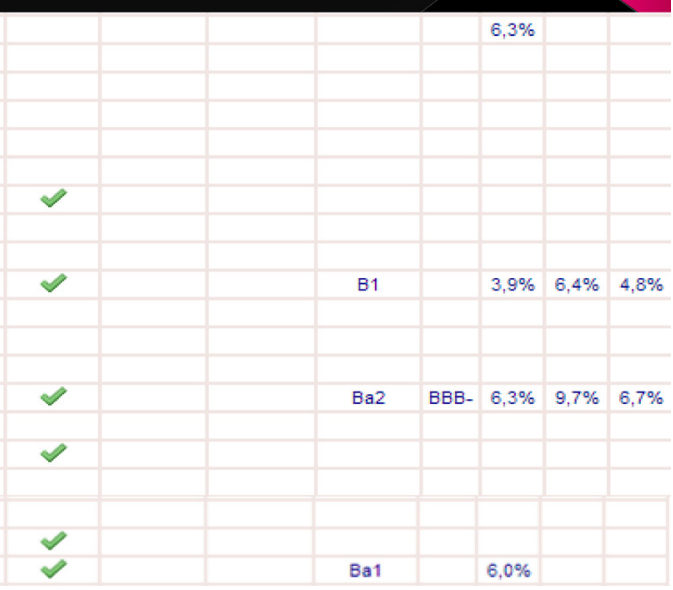




\subsubsection{Departamento económico}

El principal objetivo de las agencias es calificar los productos financieros de empresas, estados o gobiernos regionales para valorar el riesgo de impago, la solvencia del emisor o el potencial económico en general del sujeto. Las agencias de calificación afectan principalmente el mercado bursátil y financiero interno, especialmente en el sector bancario. Internacionalmente, afectan a los bonos soberanos, en el riesgo de inversión sobre las emisiones de bonos del país. Con esto un país con una prima superior acaba por pagar una mayor cantidad de dinero por cada $100 €$ de los bonos emitidos.

¿Qué supone para el conjunto de la economía? Si un país tiene una prima de 400 puntos básicos y no toma medidas, es probable que el impacto del sobrecoste por financiarse en el conjunto de su economía genere un impacto negativo en el Producto Interior Bruto de un $0,8 \%$ anual.

Las agencias pequeñas o tradicionalistas siguen el modelo basado en subscriptores, para los cuales siguen el modelo investors pay. Entidad contrata a la agencia para hacerlo obteniendo con ello un reporte privado. Las agencias de calificación entendieron que las regulaciones sobre los bonos dejaban caer que los emisores de bonos necesitaban la positividad de las agencias de calificación de riesgos para asegurar la entrada de sus bonos en los portafolios financieros.

Haciendo que estos estuvieran dispuestos a pagar por dichas calificaciones. En cuanto a la tarificación España, paga entre los 300.000 y 500.000 euros al año para que sean valoradas las emisiones de bonos sobre la deuda. Microeconómicamente la rebaja de un banco, afecta a sus instrumentos financieros y esto se extiende a sus clientes que generalmente son pequeñas y medianas empresas. Macroeconómicamente al conjunto de empresas y bancos, por ejemplo en España, la rebaja sobre la deuda española rebajo a su vez las calificaciones de las entidades financieras como el Santander, BBVA...

La declaración de ingresos de Moody's les atribuyó una ganancia bruta de 1.597.200 miles de dólares. La declaración de ingresos de McGraw Hill les atribuyó una ganancia bruta de 1.538.400 miles de dólares. McGraw Hill declaró que el 40\% de sus ingresos totales provienen de la agencia de calificación S\&P y ésta de un $14 \%$ de ese $40 \%$ del mercado. Con lo que la suma de las dos agencias hacen que el montante sea de 3.135 millones de dólares, con lo que coparían el 86\% del mercado. Con lo que la tercera agencia en discordia, es decir, Fitch, se quedaría con una couta del 14\% del mercado y un volumen de facturación de 511 millones de dólares.

Por último, explicitamos las ratios en las que basan sus calificaciones las agencias crediticias:

- I. Nivel de rentabilidad.

- 1. Utilidad Neta / Patrimonio Neto (prom.)

- 2. Utilidad Neta / Activos Totales (prom.) 
- 3. Ingreso Neto Interés + Compromiso Honorarios / Colocaciones Brutas (prom.) + Activos Líquidos (Av.) + Garantías Promedio (prom.)

- 4. No int. Exp. / Margen Financiero Rev. + Otros ingresos de explotación

- 5. Ingresos de Inversión de Capital / Inversión de Capital (Av.)

- 6. Disposición sobre los préstamos y la Parte Equidad. Garantías y / Colocaciones Brutas (prom.) + Inversión de capital (av.) y Garantías (prom.)

- II. Adecuación de capital

- 1. Generación interna de capital

- 2. Préstamos pendientes + Patrimonio Neto Invest. + Garantías netos / Capital Suscrito Reservas +

- 3. Patrimonio / Activo Total

- 4. Equidad ex. valor justo de las revalorizaciones / Activos

- 5. AAA-AA-Exigible Capital / Capital Exigible

- 6. Capital utilizable / Capital Requerido

- III. Liquidez

- 1. Activos Líquidos y de renta fija negociables Valores de Deuda / $<1$ año

- 2. Activos Líquidos y de renta fija negociables Valores / Activos totales

- 3. Activos líquidos + renta fija negociables Valores / Préstamos desembolsados y la Equidad

- IV. Calidad de activos

- 1. Préstamos deteriorados / Préstamos Brutos

- 2. Provisiones / Préstamos Brutos

- 3. Reservas Patrimoniales / Pérdida inversión de capital

- 4. Las reservas totales / Préstamos Brutos, inversiones de capital y garantías

- 5. Provisiones / No Crédito por periodificación

- 6. Los préstamos a prestatarios de grado de inversión / Préstamos Brutos Préstamos

- V. Apalancamiento

- 1. Deuda / Patrimonio

- 2. Deuda / Capital Suscrito Reservas +

- 3. Deuda / Capital Exigible 
- 4. Utilidad Neta + Intereses Pagados / Intereses Pagados.

\subsubsection{Departamento jurídico.}

Se han publicado reglamentos para regular el funcionamiento y la forma de calificación de las agencias de calificación para asegurar que sea de una forma clara y justa. Estas se han ido modificando al cabo del tiempo y perfeccionando cubriendo los vacios legales y viendo según han ido funcionando donde falla la legislación.

Estos reglamentos se han establecido a nivel europeo y España al pertenecer a la Unión Económica Europea y ratificar la normativa se ve obligada al cumplimiento de las leyes comunitarias que rige el funcionamiento de las agencias de calificación mientras no atenten contra la constitución. A parte España tiene el poder de incorporar en su legislación otras regulaciones y ampliar las existentes para su cumplimento a nivel nacional

Los reglamentos que hemos visto han sido:

- Reglamento (CE) no 1060/2009 del Parlamento Europeo y del Consejo, de 16 de septiembre de 2009, sobre las agencias de calificación crediticia.

- Reglamento delegado del 12 de julio 2012 por el que se complementa el Reglamento (CE) no 1060/2009 del Parlamento Europeo y del Consejo, de 16 de septiembre de 2009, sobre las agencias de calificación crediticia.

El Estado Español refuerza este reglamento incorporando otras leyes:

- BOE Ley 15/2011 de 16 de junio por la que se modifican determinadas normas financieras para la aplicación del Reglamento (CE) n ${ }^{\circ}$ 1060/2009 del Parlamento Europeo y del Consejo, de 16 de septiembre de 2009, sobre las agencias de calificación crediticia.

España cuenta con La comisión Nacional del Mercado y Valores cooperará con otras autoridades competentes de la UE siempre que sea necesario para llevar a cabo las funciones establecidas. En particular, intercambiará información y colaborarán en las actividades de investigación o supervisión.

El papel que tiene CNMV es el de la supervisión e inspección de los mercados de valores españoles, establece un marco regulador adaptado a las exigencias de la Unión Europea, se encarga de la protección de los inversores y velar por la transparencia y correcta formación de precios.

El Comité de Responsables Europeos de Reglamentación de Valores tiene como objetivo principal la supervisión del cumplimiento del Código de la OICV y le informara anualmente al respecto. El CERV debe mantener un registro central en el que debe guardarse información sobre el comportamiento anterior de las agencias de calificación crediticia y las calificaciones crediticias emitidas en el pasado.

La Organización Internacional de Comisiones de Valores o IOSCO) es una organización internacional que reúne a los reguladores de valores del mundo y los mercados 
de futuros. El rol principal de OICV es consigiur establecer un ambiente de trabajo adecuado para todos los miembros que intervienen en el proceso de calificación.

La IOSCO es administrada por una Secretaría General con sede en Madrid, España. Actual de la OICV Secretario General es el Sr. Greg Tanzer (ex funcionario de la Comisión de Inversiones y Valores de Australia) que es asistido por un grupo relativamente pequeño de aproximadamente 9 profesionales. ¿Cómo afecta a las agencias de calificación? Crearon un código de conducta para las agencias de calificación crediticia publicado por la Organización Internacional de Comisiones de Valores («Código de la $\mathrm{OICV} \gg)$.

La Autoridad Europea de Mercados y Valores es un comité independiente de reguladores europeos de valores establecido por la Comisión Europea el 6 de junio de 2001. Trata de mejorar la coordinación entre los reguladores de valores (tales como la CNMV, el regulador de los mercados de valores españoles) y actuar como un grupo asesor para ayudar a la Comisión Europea. También trabaja en la implementación de la legislación comunitaria en los países miembros de la UE.

\subsubsection{Departamento de imagen.}

La investigación de este departamento se centró en el estudio de las redes sociales y en los movimientos creativos que en estas se produjeron, además de un análisis exhaustivo de los perfiles de las redes sociales de las principales agencias de calificación.

\subsubsection{Moody's.}

- Facebook.

- Perfil de la organización:18 likes.

- http://www.facebook.com/\#!/pages/Moodys/230153353667538?fre$\mathrm{f}=\mathrm{ts} \& \mathrm{rf}=117505574995099$

- Perfil de grupo: 13 miembros.

- http://www.facebook.com/\#!/groups/208774445838746/?fref=ts

- No se actualiza nada, la última entrada fue posteada el 15 de enero, son empleados de la compañía y parece que posteaban noticias del sector (hablan todos en alemán con modismos y abreviaturas locales/nacionales por lo cual es imposible traducir una redacción).

- Perfil anti-Moodys: 2451 likes. Moodys Rating's stink, Moodys Ratings are junk. http://www.facebook.com/pages/Moodys-ratings-stink-moodys-ratings-are-junk/214120465298582? $\mathrm{ref}=$ ts\& $\mathrm{fref}=\mathrm{ts}$ Hablan en portugués, hablan de la crisis, critican al gobierno, los bancos y a esta agencia. Adjuntan noticias, ponen links de blogs, todo relacionado con la situación económica del mundo. 
- LinkedIn

- Bolsa de trabajo, con resumen de la empresa y detalles de empleados. http://es.linkedin.com/company/moodys-corporation?trk=tabs_biz_home

- Twitter

- 38803 tweets, 27 siguiendo, 4282 seguidores.

- Es la agencia de calificación que más utiliza el Twitter como herramienta, cada 10 minutos o menos twittean alguna noticia o actualización de calificaciones.

- Adjuntan los links de la noticia que anuncian y algunas veces ponen tweets en otro idioma que no es el inglés, pero la gran mayoría sí que está en inglés.

- https://twitter.com/MoodysRatings

- También tienen un twitter que funciona como LinkedIn, es una bolsa de trabajo. Este, lo actualizan más que LinkedIn, ya que se está interactuando todo el tiempo con posibles candidatos que también pueden escribir en su página. Como hemos dicho, el twitter es su herramienta favorita con diferencia.

- https://twitter.com/MoodysCareers

3.1.4.2. Fitch.

- Facebook

- Perfil de la organización (358 likes). http://www.facebook.com/pages/ Fitch-Ratings/114907945193379? ref $=$ ts\&fref $=$ ts

- Perfil del grupo (78 likes) http://www.facebook.com/pages/ Fitch-Ratings/114907945193379? $\mathrm{ref}=$ ts \& fref=ts\#!/pages/Fitch-Ratings/204516092953731? fref $=$ ts

- Solamente hay dos posts, adjuntando noticias, una de un Outlook negativo para la infraestructura india y otra de un chico que pide opinión acerca de otra noticia. Nada actualizado.

- No se encuentra algún grupo negativo de Fitch.

- LinkedIn

- Bolsa de trabajo, con resumen de la empresa y detalles de empleados. http://es.linkedin.com/company/fitch-ratings

- Twitter

- 2051 tweets, 492 siguiendo, 7669 seguidores https://twitter.com/FitchRatings 
- Twittean entre cada media hora y dos horas, publican más que nada noticias internas de la empresa, con quién están trabajando, a quién están analizando, y también publican muchas calificaciones de países.

\subsubsection{Standard \& Poors.}

- Facebook

- Perfil de la organización (1431 likes)

- http://www.facebook.com/pages/Fitch-Ratings/114907945193379?re$\mathrm{f}=$ ts \&fref=ts\#!/pages/Standard-poors/158580050874470? fref $=$ ts

- Perfil del grupo (82 likes)

- http://www.facebook.com/pages/FitchRatings/114907945193379?re$\mathrm{f}=$ ts\&fref=ts\#!/pages/Standard-Poors/233489220057113?fref $=$ ts

- Último post en Agosto, donde se comparte una noticia con título "La crisis podría jugar a favor de la clasificación de las economías emergentes" no se actualiza, nadie comenta, menos de 5 posts en total. Su claim en el grupo es "Rating countries to death!" Calificando países hasta la muerte.

- LinkedIn

- Bolsa de trabajo, con resumen de la empresa y detalles de empleados. http://es.linkedin.com/company/standard-\&-poor's

- Twitter

- 1784 tweets, 360 siguiendo, 23122 seguidores. https://twitter.com/standardpoors Twittean cada 2-3 horas. Publican muchos videos de noticias, hablan de previsiones de diferentes sectores para el próximo año, publican artículos de economía para entender más del sector, dejan caer preguntas importantes a los seguidores como: la capacidad de energía de Indonesia hará crecer su PIB este año, les gusta también crear polémica con sus tweets poniendo \# hash tags, no llegan a poner arrobas para que les aparezcan sus comentarios a las empresas o países comentados.

Para poder apreciar mejor estos datos presentamos unas gráficas; primero contabilizamos los likes de Facebook: 
Gráfico 13. Perfil de las organizaciones en Facebook.

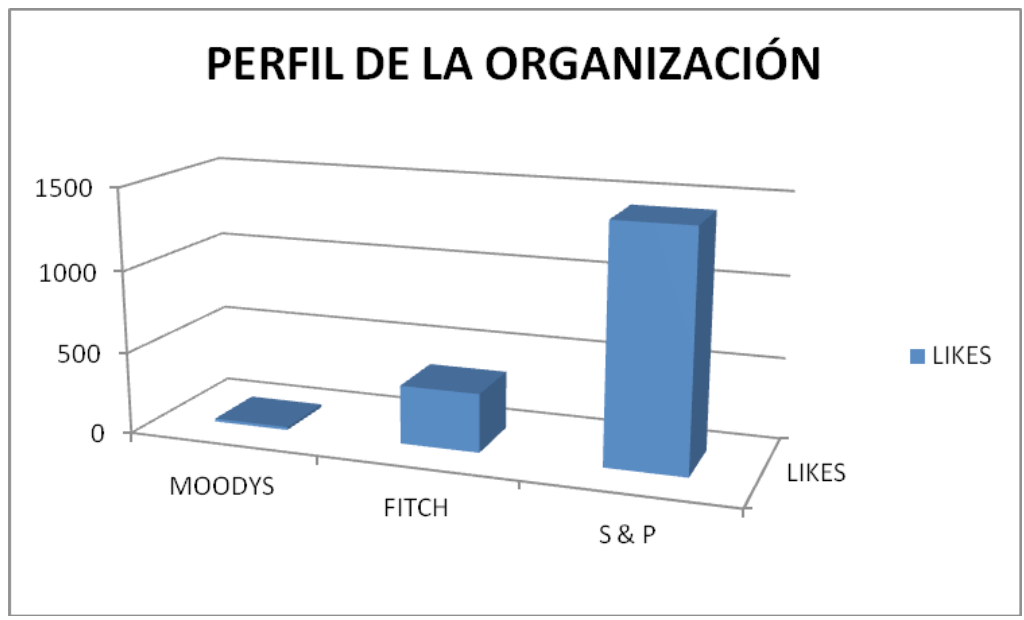

Gráfico 14. Perfil de los grupos en Facebook.

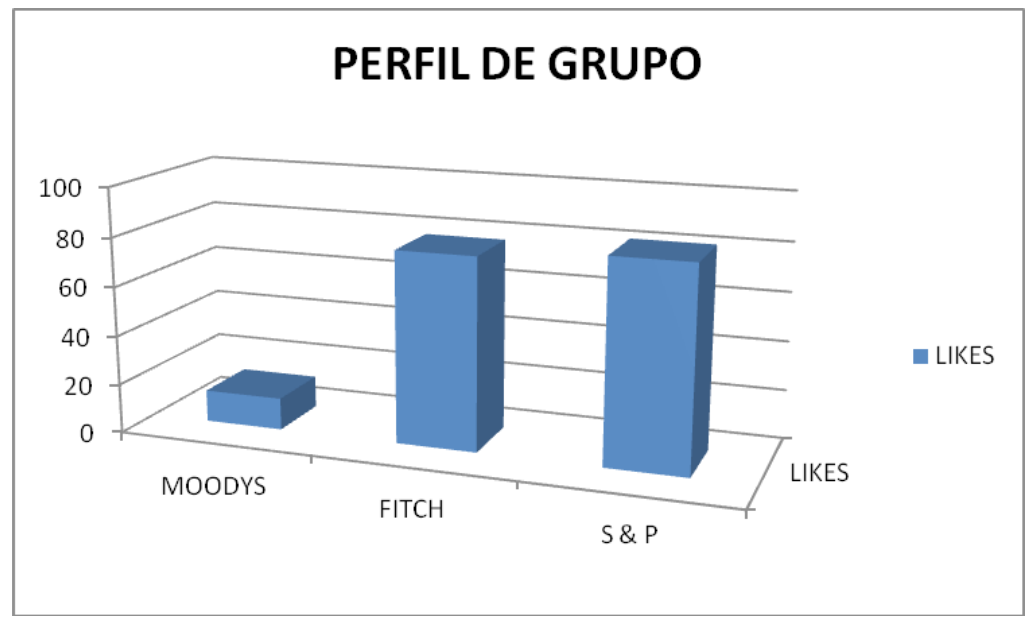

A continuación las gráficas donde podemos observar la cantidad de tweets que publica cada agencia y el número de seguidores que tiene cada una: 
Gráfico 15. Número de tweet por agencia

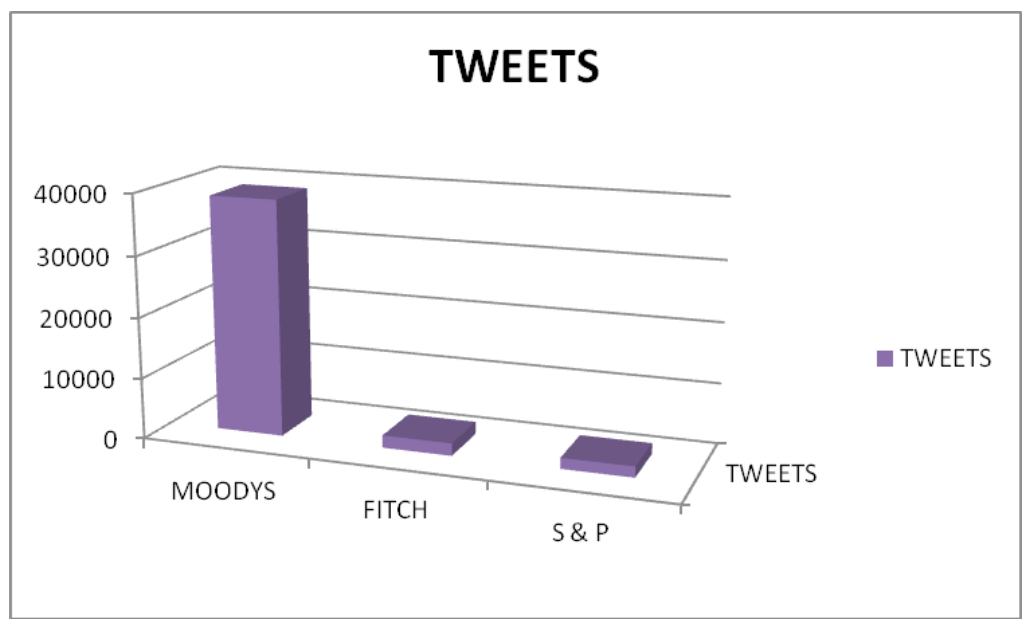

Gráfico 16. Número de followers por agencia

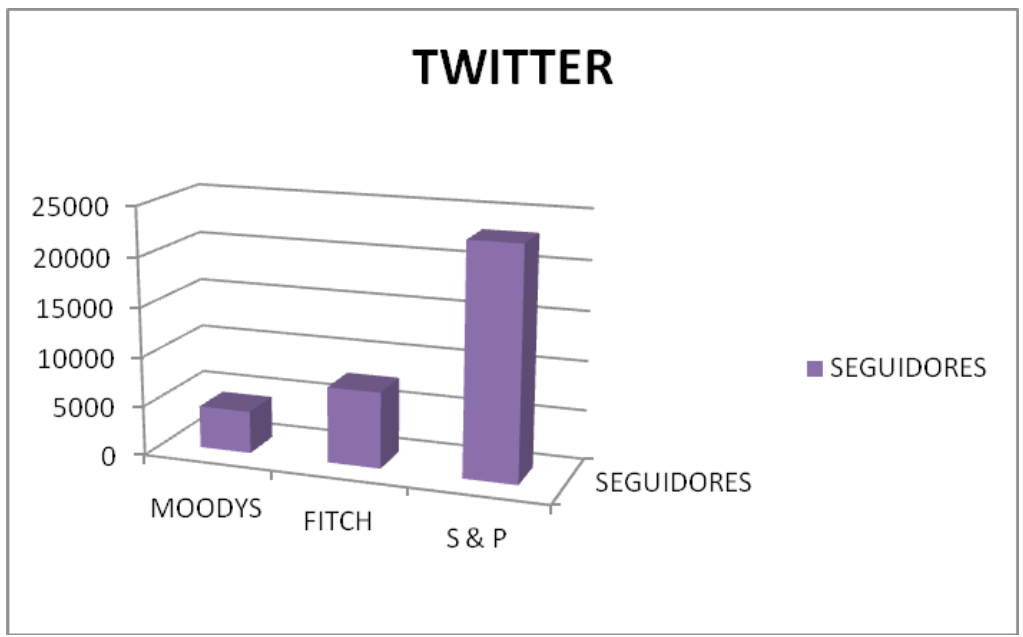

\section{Conclusiones}

En la introducción de este artículo planteábamos que el análisis que íbamos a realizar partía de una situación previa en la que las agencias de calificación no salían bien paradas en cuanto a imagen, si nos remitíamos a la opinión pública española. Pues bien, este canto de sirena debía ser contrarrestado con una búsqueda de información exhaustiva y adecuada de todo aquello que está relacionado con las agencias de cali- 
ficación. En este sentido, concluimos que se ha desarrollado correctamente el trabajo planificado con anterioridad y que, de acuerdo al marco metodológico planteado, la investigación se hallaba en buena posición para continuar su curso.

En cuanto a los criterios selectivos de información, concluimos que la división departamental ha sido adecuada, puesto que a través de los conocimientos técnicos, económicos, jurídicos y de imagen, hemos conseguido tener una visión holística, o de conjunto, de la labor que realizan las agencias de calificación en su día a día. En este sentido queremos resaltar la excelente labor realizada por los componentes de la investigación puesto que sus informes finales han permitido que la extracción de ideas fuerza haya resultado mucho más fácil tanto en calidad como en cantidad.

Es especialmente relevante el amplio y detallado trabajo que se han realizado en los departamentos técnico y de imagen. En este sentido, consideramos que la estructura de imágenes potencia los elementos presentados y ayuda al discurrir de esta fase de la investigación, lo que redundará en un mejor aprovechamiento de los grupos de presión, lobby y contralobby, toda vez que estos queden establecidos.

En el apartado técnico destacamos la presencia de multitud de variables poco o nada conocidas para el gran público u opinión pública y que ahora van a ser un elemento de empuje para el lobby de cara a la puesta en marcha de su campaña de imagen. Igualmente es valorable esta información desde el punto de vista del contralobby, puesto que se puede manejar como un elemento de distracción por parte de las propias agencias para que la opinión pública no conozca lo que realmente hacen, huyendo de los criterios de independencia y transparencia que les son propios.

Como conclusión final de esta primera fase de la campaña de imagen sobre las agencias de calificación podemos destacar el poco o nulo uso que hacen las mismas de las redes sociales y lo 2.0 y 3.0. Quitando algún perfil aislado, y despreciable desde el punto de vista de la opinión pública, estas entidades no aprovechan las potencialidades que ofrecen las nuevas tecnologías para tratar de mejorar su imagen, o que ésta no empeore cada vez más, al menos. Un fallo que también deberán aprovechar ambos grupos de presión para, una vez constituidos, ponerse a trabajar sobre ello según sean sus intereses y según perciban que se puede moldear la opinión pública en redes sociales.

\section{Webgrafía}

1. http://www.facebook.com/\#!/pages/Moodys/230153353667538?fre$\mathrm{f}=\mathrm{ts} \& \mathrm{rf}=117505574995099$

2. http://www.facebook.com/\#!/groups/208774445838746/?fref=ts

3. http://www.facebook.com/pages/Moodys-ratings-stink-moodys-ratings-are-junk/214120465298582? $\mathrm{ref}=$ ts\& fref=ts

4. http://es.linkedin.com/company/moodys-corporation?trk=tabs_biz_home

5. https://twitter.com/MoodysRatings 
6. https://twitter.com/MoodysCareers

7. http://www.facebook.com/pages/Fitch-Ratings/114907945193379? ref=ts\&fre$\mathrm{f}=\mathrm{ts}$

8. http://www.facebook.com/pages/Fitch-Ratings/114907945193379?ref=ts\&fre$\mathrm{f}=\mathrm{ts} \#$ !/pages/Fitch-Ratings/204516092953731?fref $=$ ts

9. http://es.linkedin.com/company/fitch-ratings

10. https://twitter.com/FitchRatings

11. http://www.facebook.com/pages/Fitch-Ratings/114907945193379?ref=ts\&fre$\mathrm{f}=$ ts\#!/pages/Standard-poors/158580050874470?fref $=$ ts

12. http://www.facebook.com/pages/FitchRatings/114907945193379? ref=ts\&fre$\mathrm{f}=$ ts $\#$ ! /pages/Standard-Poors/233489220057113?fref $=$ ts

13. http://es.linkedin.com/company/standard-\&-poor's

14. https://twitter.com/standardpoors 